Distribution de Pseudomonas aeruginosa et Aeromonas hydrophila dans les eaux de la nappe phréatique superficielle en zone équatoriale au Cameroun et relations avec quelques paramètres chimiques du milieu.

Distribution of Pseudomonas aeruginosa and Aeromonas hydrophila hydrophila in groundwater in equatorial zone in Cameroon and relationships with some environmental chemical factors

\author{
M. Nola, T. Njine, V. F. Sikati et E. Djuikom
}

Volume 14, numéro 1, 2001

URI : https://id.erudit.org/iderudit/705407ar

DOI : https://doi.org/10.7202/705407ar

Aller au sommaire du numéro

Éditeur(s)

Université du Québec - INRS-Eau, Terre et Environnement (INRS-ETE)

ISSN

0992-7158 (imprimé)

1718-8598 (numérique)

Découvrir la revue

\section{Citer cet article}

Nola, M., Njine, T., Sikati, V. F. \& Djuikom, E. (2001). Distribution de Pseudomonas aeruginosa et Aeromonas hydrophila dans les eaux de la nappe phréatique superficielle en zone équatoriale au Cameroun et relations avec quelques paramètres chimiques du milieu. Revue des sciences de l'eau / Journal of Water Science, 14(1), 35-53. https://doi.org/10.7202/705407ar
Résumé de l'article

Une étude microbiologique et chimique a été menée pendant un an sur les eaux de sources et de puits de Yaoundé (Cameroun). Les analyses microbiologiques ont été faites suivant la technique des membranes filtrantes, et les analyses chimiques, suivant les techniques analytiques usuelles. Les abondances maximales mensuelles de Pseudomonas aeruginosa et de Aeromonas hydrophila varient respectivement de 1 à $22 \times 10^{3}$ UFC. $100 \mathrm{ml}^{-1}$ d'eau, et de 1 à $7,8 \times 10^{3}$ UFC. $100 \mathrm{ml}^{-1}$. Ces abondances bactériennes subissent d'amples fluctuations spatio-temporelles. Les eaux de sources et de puits analysées sont faiblement bicarbonatées, douces et présentent une minéralisation faible à moyenne. Le $\mathrm{pH}$ varie de 3 à 5 et les concentrations en

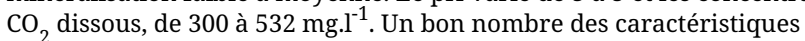
chimiques de ces eaux est relativement stable au cours du temps, en dépit des fluctuations spatiales apparentes. Le degré de corrélation entre les paramètres chimiques et la dynamique d'abondance des bactéries isolées, est hétérogène. Cela est nettement plus marqué dans les eaux de puits, en raison des conséquences de l'exploitation humaine de ces eaux, à l'origine d'apports allochtones divers. 


\title{
Distribution de Pseudomonas aeruginosa et Aeromonas hydrophila dans les eaux de la nappe phréatique superficielle en zone équatoriale au Cameroun et relations avec quelques paramètres chimiques du milieu
}

\author{
Distribution of Pseudomonas aeruginosa \\ and Aeromonas hydrophila in groundwater \\ in equatorial zone in Cameroon and relationships \\ with some environmental chemical factors
}

M. NOLA ${ }^{*}{ }^{1}$, T. NJINE $^{1}$, V.F. SIKATI ${ }^{2}$, E. DJUIKOM ${ }^{1}$

Reçu le 7 avril 2000, accepté le 15 février $2001^{\text {** }}$.

SUMMARY

\begin{abstract}
A bacteriological and chemical study was carried out on the waters of four springs and six wells over 13 months and 12 months, respectively, in the town of Yaounde and its environs in Cameroon. For these groundwater points the study analysed the importance of $\mathrm{pH}$, electrical conductivity, dissolved oxygen and carbon dioxide, and some ions such as sodium, potassium, calcium, magnesium, chloride and bicarbonate, for communities of Pseudomonas aeruginosa and Aeromonas hydrophila. The two bacteria $P$. aeruginosa and A. hydrophila are considered opportunistic pathogens. Different groundwater points were chosen on the basis of their spatial distribution and the size of the human population using them. Microbiological analyses were carried out by membrane filtration and chemical analyses were performed using standard analytical techniques.
\end{abstract}

It has been noted that the monthly maximum abundance of $P$. aeruginosa and $A$. hydrophila varies from 1 to $22 \times 10^{3} \mathrm{CFU}$ per $100 \mathrm{ml}$ and from 1 to $7.8 \times 10^{3} \mathrm{CFU}$ per $100 \mathrm{ml}$ of water, respectively. These bacteria are sometimes rare and their abundance undergoes spatio-temporal fluctuations. The studied waters are slightly bicarbonate and soft, with a low to average level of mineralisation. The $\mathrm{pH}$ varied from 3 to 5 and the concentration of dissolved $\mathrm{CO}_{2}$ from 300 to $532 \mathrm{mg} / \mathrm{l}$. Most chemical characteristics were relatively stable with time, but exhibited apparent spatial fluctuations. The level of correlation between the chemical parameters and the abundance dynamics of $P$. aeruginosa and $A$. hydrophila is heterogeneous. Depending on the origin (well or spring) of water samples and minimising their spatial variations, samples were grouped into single biotopes (either mother well or mother

1. Université de Yaoundé 1, Faculté des sciences, Laboratoire de biologie générale, BP 812,Yaoundé, Cameroun.

2. 3132 boulevard Neilson \#304, Sainte Foy, Québec, G1W 2V4, Canada.

* Correspondance. E-mail : mnola@uycdc.uninet.cm

** Les commentaires seront reçus jusqu'au 31 janvier 2002. 
spring) which were separated into compartments. A correlation test was then carried out using data obtained from 52 monthly-average samplings in the four compartments of mother-spring water, and using data obtained from 72 monthly-average samplings in the six compartments of mother-well water. From this it emerged $(\mathrm{P}<0.001)$ that in springs increases in $\mathrm{pH}$, electrical conductivity, and in concentrations of chloride, sodium, potassium, calcium, magnesium and dissolved oxygen favoured the development of $P$. aeruginosa and $A$. hydrophila. High concentrations of dissolved carbon dioxide reduced $(P<0.001)$ the abundance of these bacteria. In wells, the ecology of $P$. aeruginos $a$ and $A$. hydrophila is relatively unstable in comparison with springs. The higher instability in well water is ascribed to an increase in the number of confounding factors, which make wells appear more vulnerable than springs. This leads to the multiplication of asymmetrical interaction networks affecting bacterial population dynamics.

Key-words: P. aeruginosa, A. hydrophila, chemical parameters, tropical groundwater.

\section{RÉSUMÉ}

Une étude microbiologique et chimique a été menée pendant un an sur les eaux de sources et de puits de Yaoundé (Cameroun). Les analyses microbiologiques ont été faites suivant la technique des membranes filtrantes, et les analyses chimiques, suivant les techniques analytiques usuelles. Les abondances maximales mensuelles de Pseudomonas aeruginosa et de Aeromonas hydrophila varient respectivement de 1 à $22 \times 10^{3} \mathrm{UFC} \cdot 100 \mathrm{ml}^{-1}$ d'eau, et de 1 à $7,8 \times 10^{3} \mathrm{UFC} \cdot 100 \mathrm{ml}^{-1}$. Ces abondances bactériennes subissent d'amples fluctuations spatio-temporelles. Les eaux de sources et de puits analysées sont faiblement bicarbonatées, douces et présentent une minéralisation faible à moyenne. Le pH varie de 3 à 5 et les concentrations en $\mathrm{CO}_{2}$ dissous, de 300 à $532 \mathrm{mg} \cdot \mathrm{L}^{-1}$. Un bon nombre des caractéristiques chimiques de ces eaux est relativement stable au cours du temps, en dépit des fluctuations spatiales apparentes. Le degré de corrélation entre les paramètres chimiques et la dynamique d'abondance des bactéries isolées, est hétérogène. Cela est nettement plus marqué dans les eaux de puits, en raison des conséquences de l'exploitation humaine de ces eaux, à l'origine d'apports allochtones divers.

Mots clés : P. aeruginosa, A. hydrophila, environnement chimique, eau souterraine tropicale.

\section{1 - INTRODUCTION}

Les eaux souterraines représentent pour l'humanité une richesse vitale dont la vulnérabilité à la pollution microbienne est connue. Dans ces eaux, évoluent une faune et une microflore relativement diversifiées (BOUTIN et BOULANOUAR, 1984), la microflore stygobie étant en grande proportion bactérienne (LARPENT et LARPENT-GOURGAUD, 1990). L'environnement immédiat de ces organismes exerce sur eux des effets variables. Plusieurs communautés microbiennes vivent dans le sol et en milieux aquatiques; leur physiologie dans ces deux types de biotopes sont souvent similaires, bien que leurs sources de nutriments soient significativement différentes (ROSZAK et COLWELL, 1987). Divers facteurs sont susceptibles d'agir sur le développement des micro-organismes d'un milieu. 
En milieu aquatique, les propriétés physicochimiques et biologiques affectent significativement la survie des bactéries, l'eau jouant à la fois le rôle de " eau matière » et de « eau milieu » (FELLRATH et TARRADELLAS, 1984). Comme " eau milieu ", l'eau sert de biotope et permet le développement des microorganismes aquatiques ; comme " eau matière ", l'eau joue le rôle de solvant en même temps que celui de réactif vis-à-vis des éléments et composés chimiques présents. Ces éléments et composés chimiques dans la plupart des cas, conditionnent la survie des micro-organismes présents.

Les bactéries sont généralement les micro-organismes les plus abondants dans la nature. Leur présence non désirée dans un milieu indique un risque sanitaire plus ou moins important pour l'homme, la pathogénicité des bactéries habituellement inoffensives pouvant survenir par suite d'une immuno-dépression de l'hôte. De nombreuses bactéries qualifiées de pathogènes opportunistes, appartiennent aux genres Pseudomonas, Aeromonas, Staphylococcus, Vibrio, Legionella, Plesiomonas, Klebsiella, Serratia et Acinetobacter (LE MINOR et VÉRON, 1989 ; KREISEL, 1991). Pseudomonas aeruginosa et Aeromonas hydrophila rencontrées dans le sol, en milieux aquatiques et hospitaliers, sont responsables de diarrhées, d'infections des plaies et d'infections génito-urinaires et oculaires chez les sujets immunodéficients (LE MINOR et VÉRON, 1989). Certaines bactéries, par exemple Aeromonas salmonicida et Streptococcus uberis, présentent un risque sanitaire pour bon nombre d'animaux aquatiques (ALTMANN et al., 1992 ; CARTWRIGHT et al., 1994 ; MATTHEWS et al., 1994).

Les éléments chimiques considérés comme majeures dans les eaux souterraines sont les anions bicarbonate, carbonate, chlorure et sulfate, ainsi que les cations calcium, magnésium, potassium et sodium (BANTON et BANGOY, 1997). La nature et le degré d'influence des facteurs environnementaux sur la distribution et la survie des micro-organismes sont généralement très variés. La température du milieu, la teneur en $\mathrm{CO}_{2}$ et en matières organiques dissoutes ainsi que la nature et la concentration des nutriments disponibles peuvent affecter les taux de croissance des micro-organismes présents. Ces paramètres constituent entre autres des facteurs de variabilité de la productivité des communautés bactériennes (MARSHALL, 1988 ; WIEBE et al., 1992 ; SANDER et KALFF, 1993). Les iodures et les composés ammonium quaternaires peuvent inhiber le développement de Listeria monocytogenes, de L. ivanovii et de L. innocua (TUNCAN, 1993) sous certaines conditions environnementales. Les variations de la teneur en oxygène dissous peuvent sensiblement affecter la physiologie des bactéries métabolisant les nitrites et l'azote ammoniacal (JAYAMOHAN et al., 1988).

Peu d'études ont été menées sur la caractérisation des eaux des nappes phréatiques superficielles en zone équatoriale au Cameroun. Dans cette région, la qualité des eaux souterraines subit une grande variabilité (NOLA et al., 1998); ces eaux hébergent parfois une abondante communauté de bactéries coliformes, leur concentration subissant l'influence de la pluviométrie (NOLA et al., 1999). L'abondance des coliformes fécaux et des streptocoques fécaux dans les puits y est influencée par la profondeur totale des puits et l'épaisseur de la colonne d'eau dans ces puits (NOLA et al., 2000). Aucune action de traitement de ces ressources aquatiques n'a encore été entreprise. Peu de données relatives à l'importance des facteurs environnementaux sur le peuplement bactérien des eaux souterraines dans cette région, aux sols ferralitiques, sont disponibles. Ce travail préliminaire vise à évaluer l'importance de certaines variables chimiques sur la distribution saisonnière de $P$. aeruginosa et de 
A. hydrophila, deux bactéries pathogènes opportunistes, dans différents écosystèmes souterrains situés dans l'espace urbain de Yaoundé (Cameroun).

\section{2 - MATÉRIELS ET MÉTHODES}

\subsection{Sites de prélèvements}

Yaoundé est à $3^{\circ} 52^{\prime}$ de latitude Nord et $11^{\circ} 32^{\prime}$ de longitude Est, à une altitude moyenne de $759 \mathrm{~m}$. Cette ville au sol ferralitique est influencée par un climat équatorial classique. Quatre points d'eau de source (point de résurgence) désignés $S_{1}, S_{2}, S_{3}, S_{4}$, et six points d'eau de puits désignés $P_{1}, P_{2}, \ldots, P_{6}$, ont été choisis dans 10 quartiers différents (figure 1) sur la base de leur répartition spatiale et de leur importance relative pour les populations utilisatrices. Un point d'eau est d'autant plus important que le volume d'eau puisée est élevé et/ou que l'eau puisée est en priorité destinée à l'alimentation humaine. Tous les points d'eau de puits sont surmontés de margelles, soit en tôle, soit en béton, d'une hauteur de 0,5 à $0,8 \mathrm{~m}$ et ne sont pas régulièrement couverts. Les puits $P_{3}$ et $P_{5}$ ont des profondeurs respectives de 14 et $16 \mathrm{~m}$, alors que celles des autres puits varient de 3,5 à $8 \mathrm{~m}$. Quant aux sources sélectionnées, les eaux s'écoulent à travers les tuyaux PVC adaptés par la population. Les densités de cette population varient de 150 à 220 habitants.ha ${ }^{-1}$; ces habitants ont un taux de croissance de 5,8 à $6,2 \%$ (Directeur du recensement général de la population, communication personnelle) et utilisent pour la plupart des latrines traditionnelles. L'activité agricole dans les quartiers est pratiquée à faible échelle et sans utilisation de fertilisants. La granulométrie, la porosité et la perméabilité des sols de la région varient, respectivement, de 17 à $80 \%$, de 5 à $71 \%$ et 50 à $300 \mathrm{~cm} \cdot \mathrm{h}^{-1}$ (BACHELIER, 1959 ; HUMBEL, 1969). Le profil vertical de ces sols montre le passage de l'horizon humifère en surface à l'horizon tacheté en profondeur, en passant par l'horizon rouge argileux à pseudo-sable et l'horizon gravillonnaire (BACHELIER, 1959).

\subsection{Prélèvement et analyse des échantillons}

L'eau a été prélevée de chaque puits en utilisant un petit seau métallique de 5 litres relié à une corde métallique, les 2 ayant été préalablement stérilisés à l'autoclave. Les échantillons d'eau destinés aux analyses bactériologiques ont été recueillis dans des flacons en verre stériles de $500 \mathrm{ml}$. Les échantillons destinés aux analyses chimiques ont été prélevés dans des flacons en polyéthylène de $1000 \mathrm{ml}$. Tous ces échantillons ont été transportés au laboratoire en enceinte réfrigéré, où ils ont été immédiatement analysés.

Les analyses bactériologiques ont été faites suivant la technique des membranes filtrantes (HAVELAAR et al., 1987 ; FORD, 1994), en utilisant des membranes en ester de cellulose - Millipore, Bedford, MA 01730 - de porosité $0,45 \mu \mathrm{m}$. La recherche et l'isolement de $P$. aeruginosa ont été faits sur milieu Pyocyanosel (MARCHAL et al., 1991). L'isolement de A. hydrophila a nécessité une gélose à l'ampicilline-dextrine (HAVELAAR et al., 1987). Chaque bactérie isolée a ensuite été identifiée selon les critères biochimiques usuels, en utilisant la galerie API 20NE (Diagnostics PASTEUR, 1987 ; MARCHAL et al., 1991). 


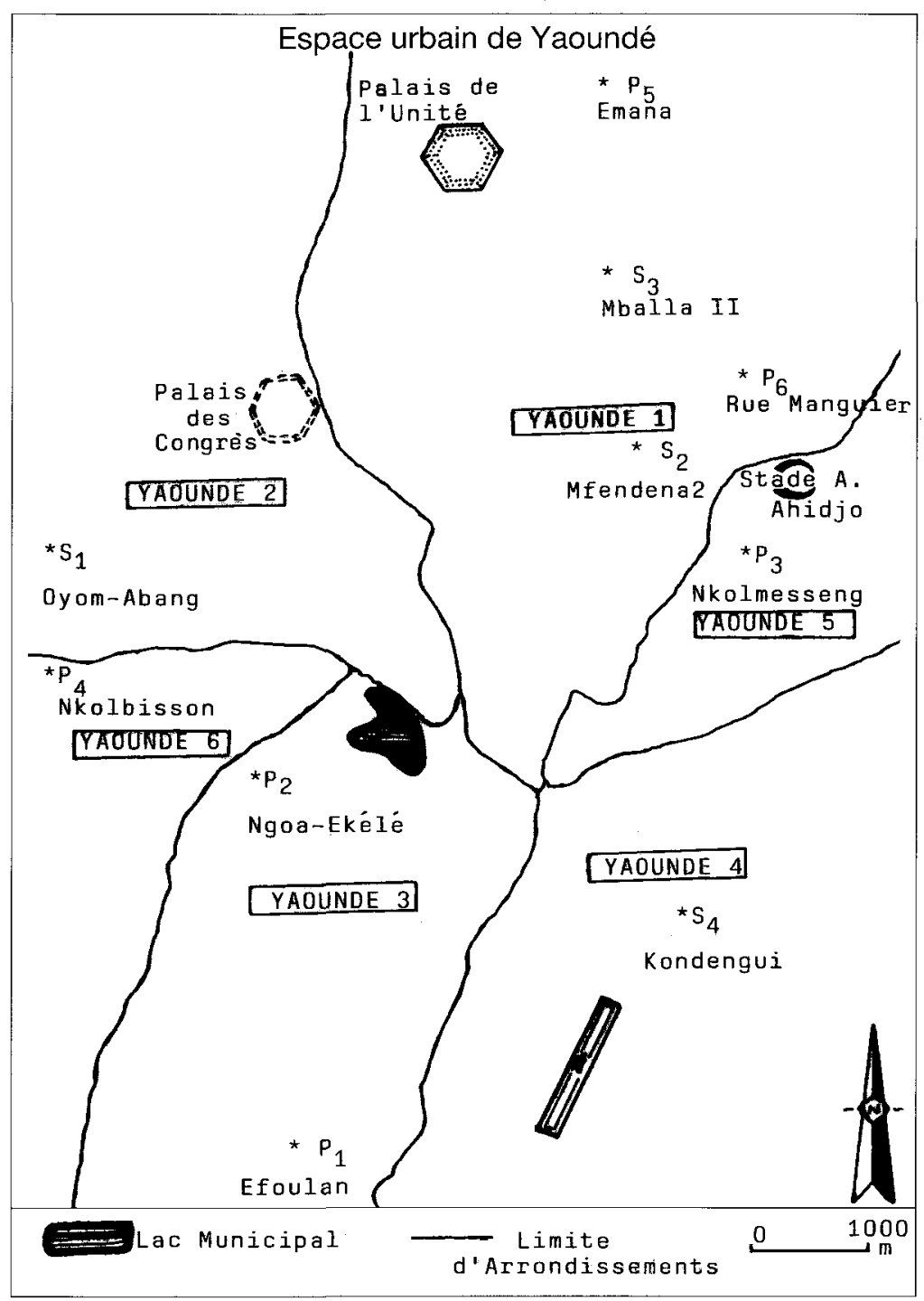

Figure 1 Emplacements des sites des prélèvements ( $S$ : point d'eau de source; $P$ : point d'eau de puits).

Location of sampling sites (S: spring water point ; $P$ : well water point).

Les principales variables chimiques analysées ont été le $\mathrm{pH}$, la conductivité électrique, le sodium, le potassium, les chlorures, le calcium, le magnésium, les bicarbonates, l'oxygène dissous et le gaz carbonique dissous. Ces analyses chimiques ont été faites suivant les techniques usuelles (APHA, 1985). Le sodium et le potassium ont ainsi été dosés par photométrie de flamme, le calcium et le magnésium par complexométrie à l'aide de l'Idranal III, les chlorures par la méthode au nitrate mercurique et les bicarbonates par la méthode à 
l'acide sulfurique. L'oxygène et le gaz carbonique dissous ont été fixés in situ et dosés au laboratoire, respectivement par la méthode de WINKLER et par la méthode à l'acide chlorhydrique. Le $\mathrm{pH}$ et la conductivité électrique ont été mesurés in situ à l'aide, respectivement, d'un pH-mètre (SCOTT Geräte CG 818) et d'un conductimètre (SCOTT Geräte CG 857).

Chaque point d'eau de source a été échantillonné tous les 7 jours, et chaque point d'eau de puits, tous les 14 jours. Cette étude a duré 13 mois pour les eaux des sources, et 12 mois pour les eaux des puits. L'étude a été réalisée du mois d'avril 1994 à avril 1995.

Les graphiques en cordonnées semi-logarithmiques ont été utilisés pour illustrer les dynamiques d'abondance des variables microbiologiques. À l'aide du test de corrélation de Spearman, les coefficients de liaison ont été calculés entre les paramètres chimiques et les paramètres microbiologiques.

\section{3 - RÉSULTATS}

Les abondances maximales mensuelles de $P$. aeruginosa varient de 1 à $22 \times 10^{3}$ UFC. $100 \mathrm{ml}^{-1}$ dans les eaux des sources, et de 1 à $1,6 \times$ $10^{3}$ UFC. $100 \mathrm{ml}^{-1}$ dans les eaux des puits ; celles de A. hydrophila dans les eaux des sources varient de 1 à $2,3 \times 10^{3}$ UFC. $100 \mathrm{ml}^{-1}$, et de 1 à $7,8 \times$ $10^{3}$ UFC $\cdot 100 \mathrm{ml}^{-1}$ dans les eaux des puits (figures 2 et 3 ). L'abondance mensuelle maximale la plus élevée de $P$. aeruginosa pour les eaux de sources a été enregistrée dans $S_{4}$ au mois de juin, et pour les eaux de puits, dans $P_{6}$ au mois d'août ; celle de $A$. hydrophila pour les eaux de sources a également été enregistrée dans $\mathrm{S}_{4}$, mais au cours du mois de novembre, et pour les eaux des puits, dans $\mathrm{P}_{3}$ au mois de mai (figures 2 et 3 ).

Dans les eaux de sources et de puits, les bactéries étudiées sont parfois rares. II en est ainsi de $P$. aeruginosa dans les sources $S_{1}$ et $S_{3}$, et dans les puits $\mathrm{P}_{2}$ et $\mathrm{P}_{4}$ (figures 2 et 3 ). La même observation est faite pour $A$. hydrophila dans les sources $S_{1}, S_{2}$ et $S_{3}$, ainsi que dans le puits $P_{4}$ (figures 2 et 3 ).

Dans les eaux de sources, le $\mathrm{pH}$ varie de 3 à 4,5 et la conductivité électrique de 22 à $175 \mu \mathrm{s} \cdot \mathrm{cm}^{-1}$. Les teneurs des ions varient de 0,3 à $17,5 \mathrm{mg} \cdot \mathrm{L}^{-1}$ pour le sodium, de 0 à $3,2 \mathrm{mg} \cdot \mathrm{L}^{-1}$ pour le potassium, de 0,8 à $13 \mathrm{mg} \cdot \mathrm{L}^{-1}$ pour les chlorures, de 1 à $18 \mathrm{mg} \cdot \mathrm{L}^{-1}$ pour le calcium, et de 0,5 à $5 \mathrm{mg} \cdot \mathrm{L}^{-1}$ pour le magnésium. Les concentrations en oxygène et en gaz carbonique dissous, varient respectivement de 0,4 à $1,5 \mathrm{mg} \cdot \mathrm{L}^{-1}$ et de 380 à $532 \mathrm{mg} \cdot \mathrm{L}^{-1}$. Les teneurs en bicarbonates varient de 0,1 à $5 \mathrm{mg} \cdot \mathrm{L}^{-1}$. Les moyennes annuelles des valeurs de ces paramètres chimiques ont été calculées pour chaque point d'eau de source et sont présentées dans le tableau 1. II en ressort que les eaux de la source $\mathrm{S}_{2}$ ont le $\mathrm{pH}$ annuel le plus élevé ; les eaux de la source $\mathrm{S}_{3}$ ont une minéralisation relativement élevée et sont les plus riches en $\mathrm{CO}_{2}$ dissous.

Dans les eaux de puits, le $\mathrm{pH}$ varie de 3 à 5 et la conductivité électrique de 15 à $250 \mu \mathrm{s} \cdot \mathrm{cm}^{-1}$. Les teneurs des ions varient de 0,6 à $40 \mathrm{mg} \cdot \mathrm{L}^{-1}$ pour le sodium, de 0 à $5,5 \mathrm{mg} \cdot \mathrm{L}^{-1}$ pour le potassium, de 1 à $10 \mathrm{mg} \cdot \mathrm{L}^{-1}$ pour le calcium, de 1,2 à $9,5 \mathrm{mg} \cdot \mathrm{L}^{-1}$ pour le magnésium, et de 0,7 à $35 \mathrm{mg} \cdot \mathrm{L}^{-1}$ pour les chlo- 

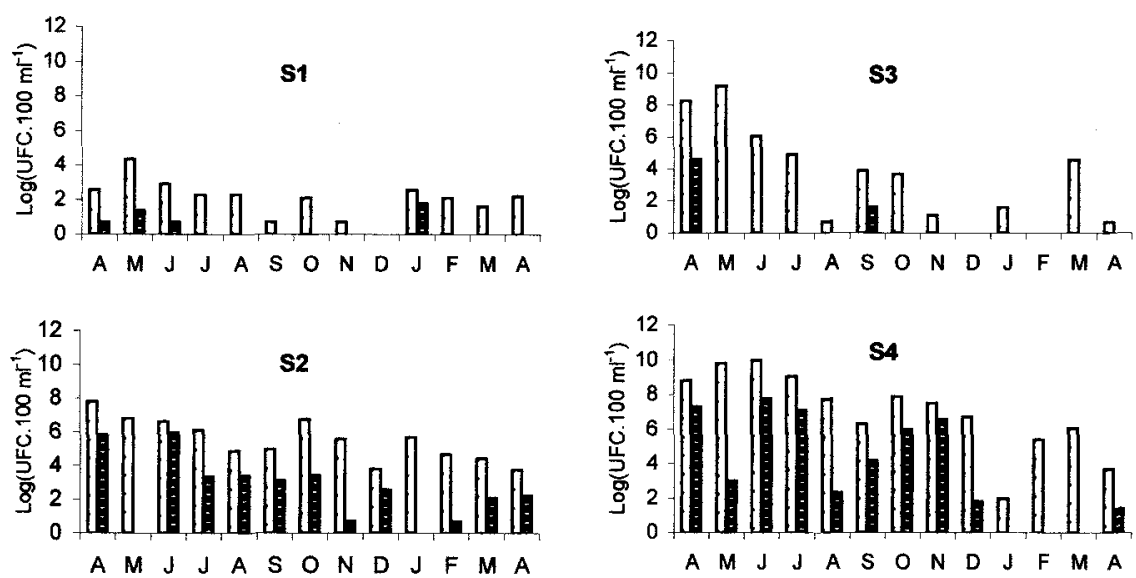

Figure 2a Variations mensuelles des densités maximales $(\square)$ et minimales $(\boldsymbol{\square})$ de $P$. aeruginosa dans les eaux des sources $S_{1}, S_{2}, S_{3}$ et $S_{4}$.

Monthly variations in the maximum densities ( $\square$ ) and minimal densities (D) of $P$. aeruginosa in springs $S_{1}, S_{2}, S_{3}$ and $S_{4}$.
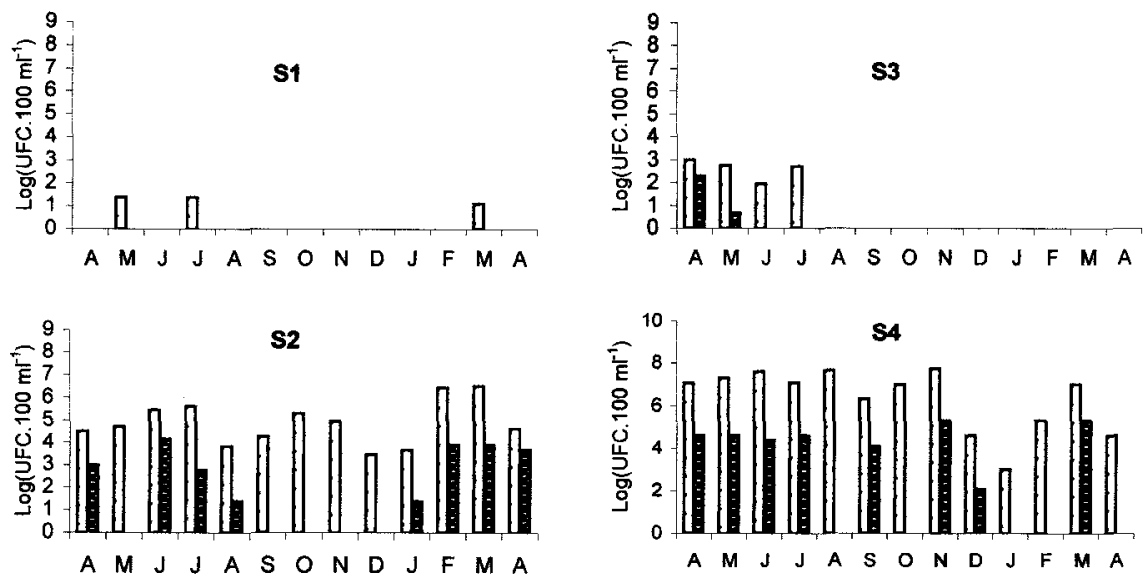

Figure 2b Variations mensuelles des densités maximales ( $\square$ ) et minimales ( $\square$ ) de A. hydrophila dans les eaux des sources $\mathrm{S}_{1}, \mathrm{~S}_{2}, \mathrm{~S}_{3}$ et $\mathrm{S}_{4}$.

Monthly variations in the maximum densities $(\square)$ and minimal densities (D) of A. hydrophila in springs $S_{1}, S_{2}, S_{3}$ and $S_{4}$.

rures. Les concentrations en $\mathrm{O}_{2}$ et en $\mathrm{CO}_{2}$ dissous varient respectivement de 0,5 à $1,2 \mathrm{mg} \cdot \mathrm{L}^{-1}$, et de 235 à $440 \mathrm{mg} \cdot \mathrm{L}^{-1}$. Les teneurs en bicarbonates varient de 0,01 à $5 \mathrm{mg} \cdot \mathrm{L}^{-1}$. Les moyennes annuelles des valeurs de ces variables chimiques, dans chaque point d'eau de puits, ont été calculées et sont présentées dans le tableau 2. 

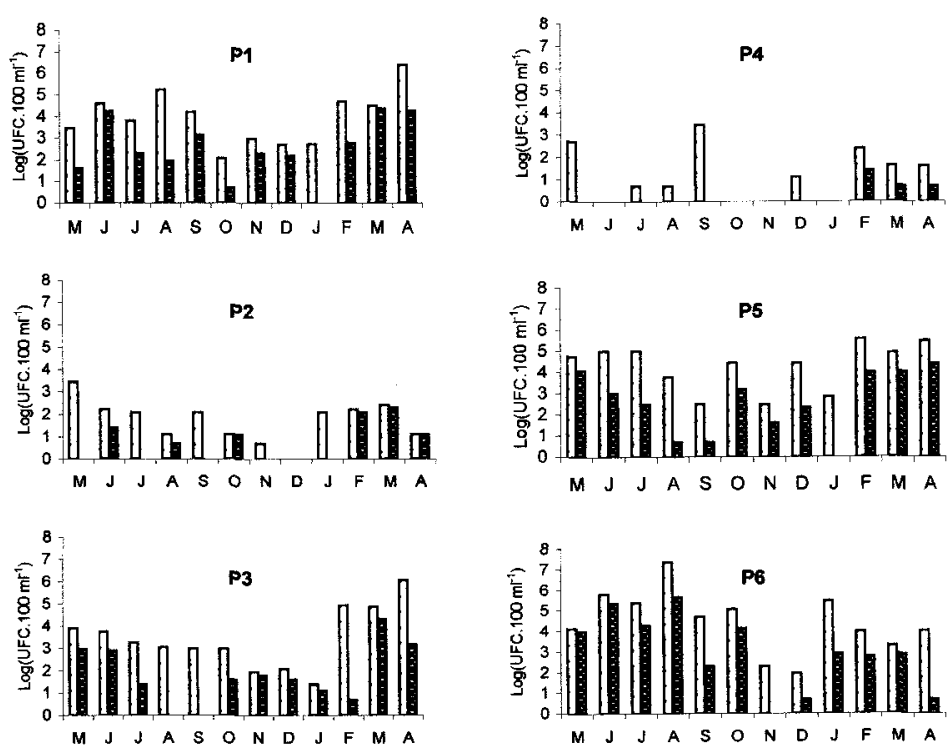

Figure 3a Variations mensuelles des densités maximales ( $\square$ ) et minimales ( $\square$ ) de $P$. aeruginosa dans les eaux des puits $\mathrm{P}_{1}, \mathrm{P}_{2}, \mathrm{P}_{3}, \mathrm{P}_{4}, \mathrm{P}_{5}$ et $\mathrm{P}_{6}$.

Monthly variations in the maximum densities $(\square)$ and minimal densities (口) of $P$. aeruginosa in wells $P_{1}, P_{2}, P_{3}, P_{4}, P_{5}$ and $P_{6}$.
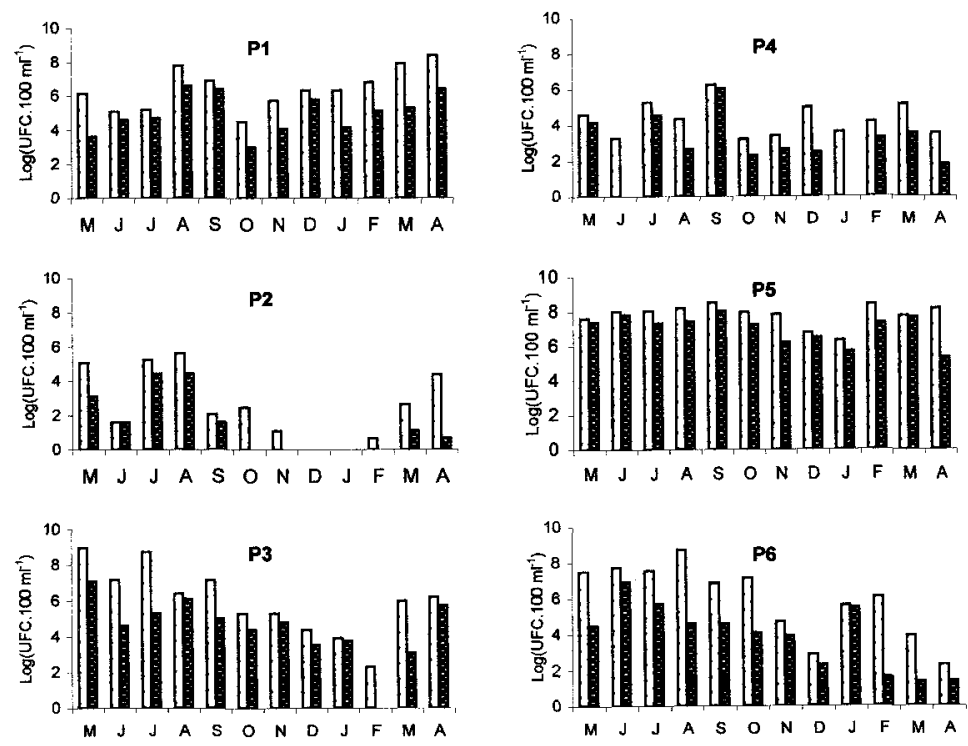

Figure 3b Variations mensuelles des densités maximales $(\square)$ et minimales ( $\square$ ) de $A$. hydrophila dans les eaux des puits $\mathrm{P}_{1}, \mathrm{P}_{2}, \mathrm{P}_{3}, \mathrm{P}_{4}, \mathrm{P}_{5}$ et $\mathrm{P}_{6}$.

Monthly variations in the maximum densities $(\square)$ and minimal densities (D) of $A$. hydrophila in wells $P_{1}, P_{2}, P_{3}, P_{4}, P_{5}$ and $P_{6}$. 
Tableau 1 Moyennes annuelles (et écart-types) des valeurs des paramètres chimiques analysées dans chaque point d'eau de source. Table 1 Annual averages and standard deviation of chemical parameters values in springs.

\begin{tabular}{|c|c|c|c|c|c|c|c|c|c|c|}
\hline $\begin{array}{l}\text { Code despH } \\
\text { sources }\end{array}$ & Cond $^{a}$ & $\begin{array}{c}\mathrm{Na}^{+} \\
\mu \mathrm{s} \cdot \mathrm{Cm}^{-1}\end{array}$ & $\begin{array}{c}\mathrm{K}^{+} \\
\mathrm{mg} \cdot \mathrm{L}^{-1}\end{array}$ & $\underset{\mathrm{mg} \cdot \mathrm{L}^{-1}}{\mathrm{Cl}^{-}}$ & $\underset{\mathrm{mg} \cdot \mathrm{L}^{-1}}{\mathrm{Ca}^{2+}}$ & $\begin{array}{c}\mathrm{Mg}^{2+} \\
\mathrm{mg} \cdot \mathrm{L}^{-1}\end{array}$ & $\begin{array}{l}\mathrm{HCO}_{3}^{-} \\
\mathrm{mg} \cdot \mathrm{L}^{-1}\end{array}$ & $\underset{\mathrm{mg}}{\mathbf{0}_{2}} \mathrm{~L}^{-1}$ & $\underset{\mathrm{mg} \cdot \mathrm{L}^{-1}}{\mathrm{CO}_{2}}$ & $\mathrm{mg} \cdot \mathrm{L}^{-1}$ \\
\hline \multirow{2}{*}{$\mathrm{S}_{1}$} & 3,46 & 25,50 & 0,78 & 0,01 & 1,24 & 1,71 & 1,00 & 1,12 & 1,11 & 439,35 \\
\hline & $(0,31)$ & $(2,98)$ & $(0,28)$ & $(0,01)$ & $(0,25)$ & $(0,37)$ & $(0,31)$ & $(1,03)$ & $(0,11)$ & $(33,79)$ \\
\hline \multirow[t]{2}{*}{$\mathrm{S}_{2}$} & 4,00 & 72,20 & 2,39 & 0,61 & 2,89 & 6,38 & 2,89 & 2,47 & 0,68 & 456,49 \\
\hline & $(0,17)$ & $(10,44)$ & $(0,52)$ & $(0,42)$ & $(0,49)$ & $(0,88)$ & $(1,28)$ & $(1,51)$ & $(0,03)$ & $(33,70)$ \\
\hline \multirow[t]{2}{*}{$\mathrm{S}_{3}$} & 3,14 & 159,13 & 14,59 & 2,26 & 11,22 & 14,11 & 2,34 & 0,42 & 0,62 & 492,55 \\
\hline & $(0,36)$ & $(10,38)$ & $(1,96)$ & $(0,53)$ & $(0,99)$ & $(2,43)$ & $(0,67)$ & $(0,28)$ & $(0,03)$ & $(31,03)$ \\
\hline \multirow[t]{2}{*}{$\mathrm{S}_{4}$} & 3,85 & 77,52 & 6,28 & 1,04 & 5,11 & 3,04 & 1,68 & 1,95 & 0,64 & 483,55 \\
\hline & $(0,19)$ & $(5,32)$ & $(0,81)$ & $(0,39)$ & $(0,41)$ & $(0,66)$ & $(0,66)$ & $(1,31)$ & $(0,03)$ & $(36,45)$ \\
\hline
\end{tabular}

$a$ : conductivité électrique 
Tableau 2 Moyennes annuelles (et écart-types) des valeurs des paramètres chimiques analysées dans chaque point d'eau de puits.

Table 2 Annual averages and standard deviation of chemical parameters in wells.

\begin{tabular}{|c|c|c|c|c|c|c|c|c|c|c|}
\hline $\begin{array}{c}\text { Code des } \\
\text { puits }\end{array}$ & $\mathbf{p H}$ & $\begin{array}{c}\mathbf{C o n d}^{a} \\
\mathbf{\mu s} \cdot \mathbf{C m}^{-1}\end{array}$ & $\begin{array}{c}\mathbf{N a}^{+} \\
\mathbf{m g} \cdot \mathbf{L}^{-1}\end{array}$ & $\begin{array}{c}\mathbf{K}^{+} \\
\mathbf{m g} \cdot \mathbf{L}^{-1}\end{array}$ & $\begin{array}{c}\mathbf{C l}^{-} \\
\mathbf{m g} \cdot \mathbf{L}^{-1}\end{array}$ & $\begin{array}{c}\mathbf{C a}^{2+} \\
\mathbf{m g} \cdot \mathbf{L}^{-1}\end{array}$ & $\begin{array}{c}\mathbf{M g}^{2+} \\
\mathbf{m g} \cdot \mathbf{L}^{-1}\end{array}$ & $\begin{array}{c}\mathbf{H C O}^{-} \\
\mathbf{m g} \cdot \mathbf{L}^{-1}\end{array}$ & $\begin{array}{c}\mathbf{O}_{2} \\
\mathbf{m g} \cdot \mathbf{L}^{-1}\end{array}$ & $\begin{array}{c}\mathbf{C O}_{2} \\
\mathbf{m g} \cdot \mathbf{L}^{-1}\end{array}$ \\
\hline $\mathrm{P}_{1}$ & 3,73 & 58,38 & 5,40 & 1,30 & 4,31 & 3,47 & 3,79 & 1,25 & 0,74 & 337,43 \\
$\mathrm{P}_{2}$ & $(0,41)$ & $(10,12)$ & $(0,94)$ & $(0,32)$ & $(1,03)$ & $(1,27)$ & $(1,02)$ & $(0,99)$ & $(0,07)$ & $(39,55)$ \\
& 3,52 & 216,29 & 32,86 & 1,59 & 31,26 & 2,44 & 2,39 & 0,72 & 0,69 & 385,13 \\
$\mathrm{P}_{3}$ & $(0,32)$ & $(28,39)$ & $(3,54)$ & $(0,34)$ & $(2,97)$ & $(0,75)$ & $(1,02)$ & $(0,48)$ & $(0,07)$ & $(34,86)$ \\
& 3,86 & 88,07 & 6,50 & 1,22 & 2,10 & 5,89 & 5,74 & 1,51 & 0,99 & 320,37 \\
$\mathrm{P}_{4}$ & $(0,32)$ & $(12,67)$ & $(1,55)$ & $(0,38)$ & $(1,91)$ & $(1,58)$ & $(1,10)$ & $(0,99)$ & $(0,09)$ & $(33,71)$ \\
& 3,08 & 86,61 & 9,09 & 1,87 & 5,41 & 2,63 & 3,85 & 0,09 & 0,77 & 314,04 \\
$\mathrm{P}_{5}$ & $(0,28)$ & $(4,39)$ & $(1,37)$ & $(0,17)$ & $(1,42)$ & $(0,43)$ & $(0,90)$ & $(0,08)$ & $(0,05)$ & $(44,75)$ \\
& 3,99 & 21,93 & 0,81 & 0,65 & 1,17 & 3,76 & 1,91 & 2,64 & 0,99 & 303,66 \\
$\mathrm{P}_{6}$ & $(0,52)$ & $(10,63)$ & $(0,19)$ & $(0,55)$ & $(0,85)$ & $(3,47)$ & $(0,79)$ & $(2,54)$ & $(0,05)$ & $(35,33)$ \\
& 3,05 & 132,46 & 17,06 & 3,39 & 10,16 & 4,19 & 4,60 & 0,26 & 0,73 & 343,69 \\
& $(0,42)$ & $(23,47)$ & $(2,90)$ & $(0,74)$ & $(2,14)$ & $(1,05)$ & $(2,10)$ & $(0,22)$ & $(0,06)$ & $(33,05)$ \\
\hline
\end{tabular}


Tableau 3 Coefficients de corrélation ( $r$ ) de Spearman entre les concentrations des paramètres chimiques et la dynamique d'abondance de $P$. aeruginosa et de $A$. hydrophila dans les eaux de source.

Table 3 Spearman correlation coefficients $(r)$ between chemical parameters and abundance dynamics of $\mathrm{P}$. aeruginosa and A. hydrophila in springs.

\begin{tabular}{|c|c|c|c|c|c|c|c|c|c|c|}
\hline \multirow{2}{*}{$\begin{array}{l}\text { Code des } \\
\text { sources }\end{array}$} & \multicolumn{2}{|c|}{$\mathrm{pH}$} & \multicolumn{2}{|c|}{ Cond ${ }^{a}$} & \multicolumn{2}{|c|}{$\mathrm{Na}^{+}$} & \multicolumn{2}{|c|}{$\mathrm{K}^{+}$} & \multicolumn{2}{|c|}{$\mathrm{Cl}^{-}$} \\
\hline & P. aerugi. & A. hydro. & P. aerugi. & A. hydro. & P. aerugi. & A. hydro. & P. aerugi. & A. hydro. & P. aerugi. & A. hydro. \\
\hline$S_{1}$ & 0,133 & $0,320^{* *}$ & 0,017 & $0,515^{\star \star \star}$ & $-0,504^{\star * *}$ & $-0,074$ & $-0,027$ & $-0,038$ & $-0,001$ & $-0,090$ \\
\hline $\mathrm{S}_{2}$ & 0,045 & $-0,040$ & $-0,483^{\star * *}$ & 0,016 & $-0,383^{\star * *}$ & $-0,020$ & $-0,486^{\star \star \star}$ & $-0,199$ & $-0,253^{\star}$ & 0,094 \\
\hline $\mathrm{S}_{3}$ & $0,289^{* *}$ & $0,347^{* * *}$ & 0,137 & $-0,320^{* *}$ & 0,137 & $-0,445^{\star \star \star}$ & $-0,368^{\star * *}$ & $-0,445^{\star \star \star}$ & $-0,029$ & 0,105 \\
\hline $\mathrm{S}_{4}$ & $0,460^{\star * *}$ & $0,570^{\star * *}$ & 0,268 & $-0,323^{\star \star}$ & 0,268 & $-0,687^{\star \star \star}$ & $-0,530^{\star * *}$ & $-0,687^{\star * *}$ & $-0,353^{\star \star \star}$ & $-0,333^{\star *}$ \\
\hline \multirow{2}{*}{$\begin{array}{l}\text { Code des } \\
\text { sources }\end{array}$} & \multicolumn{2}{|c|}{$\mathrm{Ca}^{2+}$} & \multicolumn{2}{|c|}{$\mathrm{Mg}^{2+}$} & \multicolumn{2}{|c|}{$\mathrm{HCO}_{3}^{-}$} & \multicolumn{2}{|c|}{$\mathrm{O}_{2}$} & \multicolumn{2}{|c|}{$\mathrm{CO}_{2}$} \\
\hline & P. aerugi. & A. hydro. & P. aerugi. & A. hydro. & P. aerugi. & A. hydro. & P. aerugi. & A. hydro. & P. aerugi. & A. hydro. \\
\hline $\mathrm{S}_{1}$ & $-0,145$ & 0,135 & $-0,378^{* * *}$ & $-0,003$ & $-0,006$ & 0,092 & 0,034 & $-0,098$ & $0,584^{\star \star *}$ & $0,515^{\star \star \star}$ \\
\hline $\mathrm{S}_{2}$ & $-0,112$ & $-0,195$ & $-0,273^{\star \star}$ & 0,210 & $-0,022$ & 0,043 & 0,002 & 0,087 & $-0,103$ & $-0,097$ \\
\hline $\mathrm{S}_{3}$ & $-0,105$ & $-0,001$ & $-0,248^{\star}$ & $-0,198$ & $0,620 * * *$ & 0,155 & $-0,147$ & $-0,147$ & $0,446^{* * *}$ & $-0,133$ \\
\hline $\mathrm{S}_{4}$ & $-0,102$ & $-0,101$ & 0,007 & 0,107 & 0,096 & 0,146 & $0,334^{* *}$ & $0,337^{* *}$ & $0,313^{* *}$ & 0,117 \\
\hline
\end{tabular}

a: conductivité électrique ; P. aerugi. : Pseudomonas aeruginosa ; A. hydro. : Aeromonas hydrophila

$n=56$ observations ; ${ }^{*}: P<0,1$ pour $(0,223 \leq|r|<0,264) ;{ }^{* *}: P<0,05$ pour $(0,264 \leq|r|<0,342) ;{ }^{* *}: P<0,01$ pour $(|r| \geq 0,342)$. 
Le test de corrélation de Spearman pour les données des 56 échantillons de chaque point d'eau de source et les 26 échantillons de chaque point d'eau de puits, indique que le degré de liaison entre la dynamique de chaque espèce bactérienne et l'évolution de la concentration de chaque paramètre chimique analysé est hétérogène (tableaux 3 et 4). Le pH semble favoriser $(\mathrm{P}<0,05)$ le développement de $P$. aeruginosa et de $A$. hydrophila dans les sources $\mathrm{S}_{3}$ et $\mathrm{S}_{4}$, et dans le puits $\mathrm{P}_{4}$; on note en revanche que l'augmentation du $\mathrm{pH}$ dans le puits $\mathrm{P}_{2}$ diminue la densité de $P$. aeruginosa (tableaux 3 et 4).

L'augmentation de la teneur en ion potassium diminue $(P<0,05)$ l'abondance de $P$. aeruginosa et de $A$. hydrophila dans les sources $\mathrm{S}_{3}$ et $\mathrm{S}_{4}$ (tableau 3). Dans le puits $P_{1}$, l'augmentation de la conductivité électrique, des teneurs en chlorures, en calcium et en bicarbonates semble favoriser $(P<0,05)$ le peuplement de $P$. aeruginosa et de $A$. hydrophila (tableau 4). II en est de même pour les chlorures dans la source $S_{4}$ et pour le $C_{2}$ dans la source $S_{1}$. Par contre, le sodium et le potassium semble plutôt inhiber le développement des 2 espèces bactériennes, respectivement, dans les puits $P_{2}$ et $P_{6}$ (tableau 4).

En considérant l'origine (puits ou source) des échantillons d'eau et en minimisant leurs variations spatiales, tous les échantillons des eaux de sources ont été regroupés sous forme d'un biotope qualifié de Source-Mère, et tous les échantillons des eaux de puits sous forme d'un biotope Puits-Mère. Le test de corrélation de Spearman a ensuite été appliqué aux valeurs moyennes mensuelles des données de chaque biotope Mère. Le biotope Source-Mère a ainsi 52 échantillons (52 échantillons moyens mensuels au total, réalisés dans les 4 compartiments pendant les 13 mois d'étude), et le biotope Puits-Mère, 72 échantillons (72 échantillons moyens mensuels au total, réalisés dans les 6 compartiments pendant les 12 mois d'étude). II ressort de cette approche, une autre vision des relations entre l'abondance des micro-organismes isolés et l'évolution des facteurs environnementaux pris en considération (tableau 5). La combinaison des données provenant des 4 points d'eau de source montre qu'à l'exception du $\mathrm{CO}_{2}$ dissous, qui les affecte négativement, tous les autres paramètres analysés favorisent à des degrés divers, les populations de $P$. aeruginosa et de $A$. hydrophila (tableau 5). En ce qui concerne les eaux de puits, la combinaison de toutes les données montre que la nature et le degré des relations entre $P$. aeruginosa ou $A$. hydrophila et les paramètres chimiques analysés, varient plus fortement que pour les eaux de source, $A$. hydrophila étant nettement plus sensible aux facteurs chimiques dans les puits que $P$. aeruginosa (tableau 5).

\section{4 - DISCUSSION}

Les eaux des sources et des puits analysées hébergent les communautés de $P$. aeruginosa et de $A$. hydrophila. Les densités de ces 2 bactéries pathogènes opportunistes subissent des fluctuations spatio-temporelles. Dans cette région, la concentration des bactéries coliformes dans les eaux souterraines atteint parfois $9 \times 10^{5}$ UFC. $100 \mathrm{ml}^{-1}$ (NOLA et al., 1999). Les fluctuations observées seraient liées aux conditions de l'environnement. L'eau d'une nappe 
Tableau 4 Coefficients de corrélation ( $r$ ) de Spearman entre les concentrations des paramètres chimiques et la dynamique d'abondance de $P$. aeruginosa et de $A$. hydrophila dans les eaux de puits.

Table 4 Spearman correlation coefficients ( $r$ ) between chemical parameters and abundance dynamics of $\mathrm{P}$. aeruginosa and A. hydrophila in wells.

\begin{tabular}{|c|c|c|c|c|c|c|c|c|c|c|}
\hline \multirow{2}{*}{$\begin{array}{l}\text { Code des } \\
\text { sources }\end{array}$} & \multicolumn{2}{|c|}{$\mathrm{pH}$} & \multicolumn{2}{|c|}{ Cond $^{a}$} & \multicolumn{2}{|c|}{$\mathrm{Na}^{+}$} & \multicolumn{2}{|c|}{$\mathrm{K}^{+}$} & \multicolumn{2}{|c|}{$\mathrm{Cl}^{-}$} \\
\hline & P. aerugi. & A. hydro. & P. aerugi. & A. hydro. & P. aerugi. & A. hydro. & P. aerugi. & A. hydro. & P. aerugi. & A. hydro. \\
\hline$P_{1}$ & 0,178 & $0,433^{\star \star}$ & $0,629^{\star \star \star}$ & $0,583^{\star \star \star}$ & 0,151 & 0,127 & 0,085 & 0,053 & $0,511^{\star *}$ & $0,467^{\star \star *}$ \\
\hline$P_{2}$ & $-0,439 * *$ & 0,229 & $-0,597^{\star *}$ & $-0,281$ & $-0,452^{* *}$ & $-0,426^{* *}$ & $-0,028$ & $-0,022$ & $-0,223$ & $-0,381^{*}$ \\
\hline $\mathrm{P}_{3}$ & $-0,022$ & $-0,072$ & $0,754^{* \star *}$ & $-0,098$ & 0,022 & $-0,117$ & $0,792^{* \star \star}$ & 0,273 & 0,277 & $-0,016$ \\
\hline $\mathrm{P}_{4}$ & $0,526^{\star \star \star}$ & $0,580^{\star \star \star}$ & $-0,216$ & $-0,238$ & $-0,187$ & $-0,213$ & 0,197 & 0,242 & $-0,220$ & $-0,241$ \\
\hline$P_{5}$ & $-0,178$ & 0,050 & $0,400^{\star *}$ & 0,019 & $-0,310$ & $-0,004$ & $-0,033$ & $0,369^{*}$ & $-0,185$ & $0,439^{\star *}$ \\
\hline$P_{6}$ & $-0,107$ & $-0,072$ & $-0,373^{*}$ & $0,538^{* \star *}$ & $-0,184$ & $-0,260$ & $-0,339^{\star}$ & $-0,435^{\star \star}$ & $-0,227$ & $-0,370^{*}$ \\
\hline \multirow{2}{*}{$\begin{array}{l}\text { Code des } \\
\text { sources }\end{array}$} & \multicolumn{2}{|c|}{$\mathrm{Ca}^{2+}$} & \multicolumn{2}{|c|}{$\mathrm{Mg}^{2+}$} & \multicolumn{2}{|c|}{$\mathrm{HCO}_{3}^{-}$} & \multicolumn{2}{|c|}{$\mathrm{O}_{2}$} & \multicolumn{2}{|c|}{$\mathrm{CO}_{2}$} \\
\hline & P. aerugi. & A. hydro. & P. aerugi. & A. hydro. & P. aerugi. & A. hydro. & P. aerugi. & A. hydro. & P. aerugi. & A. hydro. \\
\hline$P_{1}$ & $0,564^{* * *}$ & 0,329 & $-0,207$ & $-0,113$ & $0,351^{*}$ & $0,497^{\star \star \star}$ & 0,015 & 0,080 & $-0,103$ & $-0,179$ \\
\hline$P_{2}$ & $-0,003$ & $-0,072$ & $-0,013$ & $-0,046$ & $-0,338^{\star}$ & $0,394^{* *}$ & $-0,317$ & $-0,020$ & 0,310 & $0,337^{*}$ \\
\hline $\mathrm{P}_{3}$ & 0,277 & 0,013 & $-0,295$ & 0,273 & 0,048 & $-0,116$ & $-0,439^{\star \star}$ & $-0,119$ & $-0,021$ & $0,581^{* * *}$ \\
\hline $\mathrm{P}_{4}$ & $0,556^{\star * \star}$ & $0,411^{* *}$ & $0,355^{\star}$ & 0,246 & $0,454^{\star *}$ & $0,661^{* * *}$ & $0,439^{* *}$ & $0,399^{* *}$ & 0,103 & 0,080 \\
\hline$P_{5}$ & $0,381^{*}$ & 0,050 & $-0,350^{*}$ & $-0,447^{\star *}$ & $-0,074$ & 0,182 & 0,073 & $-0,062$ & $-0,021$ & 0,053 \\
\hline$P_{6}$ & $-0,105$ & $-0,249$ & $-0,261$ & $-0,204$ & $0,338^{\star}$ & 0,258 & 0,024 & $-0,010$ & 0,012 & 0,102 \\
\hline
\end{tabular}

a : conductivité électrique ; P. aerugi. : Pseudomonas aeruginosa ; A. hydro. : Aeromonas hydrophila

$\mathrm{n}=26$ observations ; ${ }^{*}: \mathrm{P}<0,1$ pour $(0,331 \leq|\mathrm{r}|<0,389) ;{ }^{\star \star}: \mathrm{P}<0,05$ pour $(0,389 \leq|\mathrm{r}|<0,497) ;{ }^{\star \star \star}: \mathrm{P}<0,01$ pour $(|\mathrm{r}| \geq 0,497)$ 
Tableau 5 Coefficients de corrélation ( $r$ ) de Spearman entre les moyennes mensuelles des concentrations des paramètres chimiques et les moyennes mensuelles des densités de $P$. aeruginosa et de A. hydrophila, isolées, d'une part dans les eaux des sources, et d'autre part dans les eaux des puits.

Table 5 Spearman correlation coefficients $(r)$ between the monthly averages of both water chemical parameters and abundance of $\mathrm{P}$. aeruginosa et de A. hydrophila isolated in springs and in wells.

\begin{tabular}{|c|c|c|c|c|c|c|c|c|c|c|}
\hline \multirow{2}{*}{$\begin{array}{l}\text { Origine des } \\
\text { échantillons }\end{array}$} & \multicolumn{2}{|c|}{$\mathrm{pH}$} & \multicolumn{2}{|c|}{ Cond $^{a}$} & \multicolumn{2}{|c|}{$\mathrm{Na}^{+}$} & \multicolumn{2}{|c|}{$\mathbf{K}^{+}$} & \multicolumn{2}{|c|}{$\mathrm{Cl}^{-}$} \\
\hline & P. aerugi. & A. hydro. & P. aerugi. & A. hydro. & P. aerugi. & A. hydro. & P. aerugi. & A. hydro. & P. aerugi. & A. hydro. \\
\hline Sources" & $0,788^{* *}$ & $0,672^{* *}$ & $0,793^{\star *}$ & $0,635^{\star \star}$ & $0,804^{* *}$ & $0,521^{\star \star}$ & 0,760 ** & $0,620^{* *}$ & $0,824^{* *}$ & $0,637^{\star \star}$ \\
\hline Puits $\$$ & 0,253 & $0,510^{* *}$ & 0,262 & $-0,284$ & 0,117 & $-0,354^{*}$ & $0,310^{\star}$ & 0,141 & 0,227 & $-0,341^{*}$ \\
\hline \multirow{2}{*}{$\begin{array}{l}\text { Origine des } \\
\text { échantillons }\end{array}$} & \multicolumn{2}{|c|}{$\mathrm{Ca}^{2+}$} & \multicolumn{2}{|c|}{$\mathrm{Mg}^{2+}$} & \multicolumn{2}{|c|}{$\mathrm{HCO}_{3}^{-}$} & \multicolumn{2}{|c|}{$\mathrm{D}_{2}$} & \multicolumn{2}{|c|}{$\mathrm{CO}_{2}$} \\
\hline & P. aerugi. & A. hydro. & P. aerugi. & A. hydro. & P. aerugi. & A. hydro. & P. aerugi. & A. hydro. & P. aerugi. & A. hydro. \\
\hline Sources" & $0,679^{\star \star}$ & $0,848^{* *}$ & $0,678^{* *}$ & 0,252 & $0,592^{\star *}$ & $0,776^{\star \star *}$ & $0,580^{* *}$ & $0,475^{\star *}$ & $-0,594^{\star *}$ & $-0,437^{\star}$ \\
\hline Puits $§$ & 0,278 & 0,257 & 0,117 & 0,015 & 0,074 & $0,599^{\star \star \star}$ & $-0,168$ & $0,485^{\star \star}$ & $-0,096$ & 0,138 \\
\hline
\end{tabular}

a : conductivité électrique ; P. aerugi. : Pseudomonas aeruginosa; A. hydro. : Aeromonas hydrophila

\# $: \mathrm{n}=52$ observations ; ${ }^{*}: \mathrm{P}<0,01$ pour $(0,354 \leq|\mathrm{r}|<0,443) ;{ }^{* *}: \mathrm{P}<0,001$ pour $(|\mathrm{r}| \geq 0,443)$

$\$: n=72$ observations ; ${ }^{*}: P<0,01$ pour $(0,302 \leq|r|<0,380) ;{ }^{\star \star}: P<0,001$ pour $(|r| \geq 0,380)$ 
phréatique apparaît d'autant plus vulnérable à la pollution microbiologique que le toit de la nappe est proche de la surface du sol et que les terrains qui surmontent l'aquifère sont perméables aux sources superficielles de pollution (BOUTIN, 1987).

La nature acide des biotopes impliqués dans cette étude serait liée au sol encaissant qui a un pH acide (YONGUE-FOUATEU, 1986). En considérant séparément chaque biotope, le $\mathrm{pH}$ semble influencer $(\mathrm{P}<0,05)$ la dynamique d'abondance de $P$. aeruginosa ou de $A$. hydrophila, que dans peu de sites (tableaux 3 et 4). $P$. aeruginosa est préférentiellement neutrophile et $A$. hydrophila, neutrophile à légèrement basophile (LE MINOR et VERON, 1989). L'action indirecte du $\mathrm{pH}$ se fait notamment par la modification du coefficient d'assimilation des différents composés nutritifs minéraux ou organiques par les bactéries, dont l'importance va dépendre de la tolérance des bactéries vis-à-vis de l'acidité du milieu (DOMMERGUES et MANGENOT, 1970). $A$. hydrophila et $P$. aeruginosa auraient développé dans les points d'eau étudiés, des stratégies pour minimiser l'effet de l'acidité du milieu. Ces stratégies peuvent parfois consister en la création d'agrégats bactériens qui supportent mieux les $\mathrm{pH}$ acides que les cellules isolées (DE BOER et al., 1991). Certaines bactéries vivant en milieu acide peuvent développer une résistance par l'induction de la synthèse de protéines protectrices (CHEVILLE et al., 1996). L'inactivation parfois observée de Escherichia coli, $A$. hydrophila, $P$. aeruginosa ou de Vibrio parahaemolyticus dans les milieux acides peut ne pas dépendre directement $\mathrm{du} \mathrm{pH}$, mais de la sévérité des conditions générales régnant dans ces milieux, tels que la température, la présence ou la concentration de certains sels (ENTANI et al., 1997). Une modélisation mathématique de l'inactivation de Salmonella typhimurium, de Staphylococcus aureus et Vibrio parahaemolyticus en milieu acide acétique a montré que l'effet antibactérien du faible $\mathrm{pH}$ est renforcé par l'addition du $\mathrm{NaCl}$ dans le milieu (TSUJIHATA et al., 1998).

La nature et le degré des relations entre l'abondance de $P$. aeruginosa ou celle de $A$. hydrophila et les teneurs en $\mathrm{Na}^{+}, \mathrm{K}^{+}, \mathrm{Cl}^{-}, \mathrm{Ca}^{2+}$ et $\mathrm{Mg}^{2+}$, paraissent endogènes aux biotopes étudiés (tableaux 3 et 4 ). II est connu que les espèces du genre Aeromonas n'exigent que de traces de $\mathrm{NaCl}$ pour leur croissance optimale (LE MINOR et VÉRON, 1989). Les ions influencent aussi l'adhésion des micro-organismes dans un milieu. L'adhésion de Azotobacter aux cations est ainsi plus élevée en milieu saturé en $\mathrm{Fe}^{3+}$ et $\mathrm{Al}^{3+}$, qu'en milieu saturé en $\mathrm{Na}^{+}$et $\mathrm{K}^{+}$, cette adhésion étant en général favorisée par les $\mathrm{pH}$ acides (DOMMERGUES et MANGENOT, 1970). Des études en milieu eutrophe ont montré que la hausse de la conductivité électrique favorise la colonisation du milieu par $A$. hydrophila (BURTON et LANZA, 1987). L'augmentation de la concentration en sels dans un milieu peut aussi y minimiser l'inhibition par irradiation, de certains bacilles comme Escherichia coli (FUJIKAWA et al., 1992).

Les ions $\mathrm{Ca}^{2+}$ ou $\mathrm{Mg}^{2+}$ semblent dans certains points d'eau défavoriser le développement de $P$. aeruginosa ou de $A$. hydrophila (tableaux 3 et 4). Ces ions interviennent en général dans de nombreux processus métaboliques de la bactérie et sont nécessaires à l'intégrité physique de divers constituants des micro-organismes (PELMONT, 1993).

En combinant tous les échantillons d'eau de source en un seul biotope Source-Mère et tous les échantillons d'eau de puits en un seul biotope PuitsMère, on constate que les paramètres chimiques analysés influencent l'abon- 
dance de $P$. aeruginosa et de $A$. hydrophila différemment dans les 2 types de biotopes étudiés (tableau 5).

Dans les eaux de source, les grandes concentrations de $\mathrm{CO}_{2}$ dissous défavorisent le développement de $P$. aeruginosa et de $A$. hydrophila (tableau 5). WILLOCX et al. (1993) ont noté que la sensibilité au $\mathrm{CO}_{2}$ varie suivant les espèces bactériennes. De faibles concentrations de $\mathrm{CO}_{2}$ du milieu (concentrations inférieures à $300 \mathrm{ppm}$ ) stimulent le développement de plusieurs espèces bactériennes, comme $P$. fluorescens. À forte concentration, le $\mathrm{CO}_{2}$ devient inhibiteur pour la bactérie. Les fortes concentrations de $\mathrm{CO}_{2}$ dans le milieu augmentent le temps de latence et le temps de génération et donc, diminuent le taux de croissance et le niveau de plateau chez $P$. fluorescens (WILLOCX et al., 1993). L'accroissement de l'influence de $\mathrm{CO}_{2}$, souvent liée à de faibles températures (WILLOCX et al., 1993), serait dû à la grande solubilité de ce gaz à de telles températures (RODIER, 1996). Certaines bactéries, notamment $P$. fragi, Bacillus cereus et Streptococcus cremoris, peuvent néanmoins développer une résistance aux fortes teneurs du milieu en $\mathrm{CO}_{2}$ (ENFORS et MOLIN, 1980). La survie des bactéries dans les eaux souterraines de Yaoundé à fortes concentrations en $\mathrm{CO}_{2}$ dissous serait, sans doute, liée à des adaptations physiologiques particulières.

La différence entre l'influence des facteurs environnementaux dans le biotope Source-Mère et dans le biotope Puits-Mère, résulterait de la grande et variable vulnérabilité des eaux de puits par rapport aux eaux de sources. Dans les eaux de puits, outre les apports externes des populations utilisatrices, les mouvements de la colonne d'eau lors des puisages occasionneraient une érosion de la paroi des puits, créant ainsi des dissolutions substantielles des minéraux du sol encaissant. Les grandeurs des paramètres chimiques des eaux de puits seraient de ce fait plus instables dans le temps, par rapport à celles des eaux de sources.

La solubilité du $\mathrm{CO}_{2}$ et de l'oxygène dans les eaux de puits est fonction de la pression partielle des gaz dans l'atmosphère, de la température de l'eau (la solubilité diminuant lorsque la température s'élève) et de la teneur en électrolytes, ceux-ci réduisant la solubilité des gaz (RODIER, 1996). Les variations spatio-temporelles des teneurs en $\mathrm{CO}_{2}$ (tableaux 1 et 2) refléteraient aussi la respiration des sols encaissants liée à la dégradation des composés organiques par les micro-organismes présents (DUCHAUFOUR, 1997). II est indiqué que les faibles teneurs en $\mathrm{O}_{2}$ dissous renforcent l'effet inhibiteur du $\mathrm{CO}_{2}$ du milieu (DIXON et al., 1987), les très fortes teneurs pouvant parfois à leur tour défavoriser le développement de $A$. hydrophila en milieu aquatique superficiel (SEIDLER et al., 1980 ; KAPER et al., 1981).

Des études antérieures ont suggéré que la qualité générale des eaux souterraines de Yaoundé est assujettie à une grande variabilité dont les facteurs prépondérants peuvent être, selon les sites, soit à dominance abiotique, soit à dominance biotique (NOLA et al., 1998). La présente étude montre que dans les eaux de sources et de puits analysées, le niveau de développement des populations bactériennes étudiées serait, en réalité, déterminé par la résultante des interactions entre les facteurs physiques, chimiques et biologiques. Ces facteurs établissent ainsi par leurs actions, un réseau d'interactions complexes agissant de manière différentielle sur chaque compartiment de l'écosystème (FRONTIER, 1977). 


\section{5 - CONCLUSION}

L'augmentation du $\mathrm{pH}$, de la conductivité électrique, des teneurs en oxygène dissous, en chlorures, en sodium, en potassium et en éléments alcalinoterreux dans les eaux souterraines de Yaoundé, favorise le développement de $P$. aeruginosa et de $A$. hydrophila. En revanche, les fortes teneurs en $\mathrm{CO}_{2}$ dissous diminuent la densité de ces bactéries. Toutefois, dans les eaux de puits, les réseaux d'interactions sont apparemment plus complexes que dans les eaux de source, en raison des différences notables dans l'exploitation humaine de ces 2 types de systèmes hydriques phréatiques.

\section{RÉFÉRENCES BIBLIOGRAPHIQUES}

ALTMANN K., MARSHALL M., NICHOLSON S.E., HANNA P.J., GUDKOVS N., 1992. Glucose repression of pigment production in atypical isolates of Aeromonas salmonicida responsible for goldfish ulcer disease. Microbios, 72, 215-220.

APHA-AWWA-WPCF, 1985. Standard methods for the examination of water and waste water, $16^{\text {th }}$ edition, APHA, Washington DC.

BACHELIER G., 1959. Étude pédologique des sols de Yaoundé : contribution à l'étude de la pédogenèse des sols ferralitiques. L'agronomie Tropicale, 14, 279305.

BANTON O., BANGOY L.M., 1997. Hydrogéologie, multiscience environnementale des eaux souterraines, PUQ-AUPELF, Sainte-Foy.

BOUTIN C., 1987. L'eau des nappes phréatiques superficielles, une richesse naturelle vitale mais vulnérable. L'exemple des zones rurales du Maroc. Sciences de l'Eau, 6, 357-365.

BOUTIN C., BOULANOUAR M.,1984. Premières données sur la faune des puits des environs de Marrakech (Maroc Occidental). Verh. Internat. Verein. Limnol., $22,1762-1765$.

BURTON G.A., LANZA G.R., 1987. Aeromonas hydrophila densities in thermallyaltered reservoir water and sediments. Water, Air and Soil Pollution, 34, 199206.
CARTWRIGHT G.A., CHEN D., HANNA P.J., GUDKOVS N., TAJIMA K., 1994. Immunidiagnosis of virulent strains of Aeromonas hydrophila associated with epizotic ulcerative syndrome (EUS) using a monoclonal antibody. J. Fish Diseases, 17, 123-133.

CHEVILLE A.M., ARNOLD K.W., BUCHRIESER C., CHENG C.M., KASPAR C.W., 1996. rpos regulation of acid, heated salt tolerance in Escherichia coli 0157: H7. Appl. Environ. Microbiol., 62, 1882-1884.

DE BOER W., KLEIN GUNNEWIEK P.J.A., VEENHUIS M., BOCK E., LAANBROEK H.J., 1991. Nitrification at low pH by aggregated chemolithotrophic bacteria. Appl. Environ. Microbiol., 57, 3600-3604.

DIAGNOSTICS PASTEUR, 1987. Milieux et réactifs de laboratoire. Microbiologie et immunologie, $3^{\ominus}$ édition, DIAGNOSTICS PASTEUR, Paris.

DIXON M.N., LOVITT R.W., KELL D.B., MORRIS J.G., 1987. Effect of $\mathrm{pCO}_{2}$ on the growth and metabolism of Clostridium sporogenes NCIB 8053 in defined media. J. Appl. Bact., 63, 171-182.

DOMMERGUES Y., MANGENOT F., 1970. Écologie microbienne du sol, MASSON, Paris.

DUCHAUFOUR P., 1997. Abrégé de pédologie - Sol, végétation, environnement, $5^{\ominus}$ édition, MASSON, Paris.

ENFORS S.O., MOLIN G., 1980. Effect of high concentrations of carbon dioxide, 
on growth rate of Pseudomonas fragi, Bacillus cereus and Streptococcus cremoris. J. Appl. Bact., 48, 409-416.

ENTANI E., ASAI M., TSUJIHATA S., TSUKAMOTO Y., OHTA M., 1997. Antibacterial action of vinegar against food-borne pathogenic bacteria including Escherichia coli 0157: H7 (part 1). Examination of bacteriostatic and bactericidal activities. Jpn. J. Ass. Inf. Dis., 71, 443-450.

FELLRATH M., TARRADELLAS D.J., 1984. Qualité des eaux. Monographie d'écologie des polluants. $2^{\mathrm{e}}$ édition, École Polytechnique Fédérale de Lausane, Institut du Génie de l'Environnement, Lausane.

FORD L.A., 1994. Detection of Aeromonas salmonicida from water using a filtration method. Aquaculture, 122, 1-7.

FRONTIER S., 1977. Réflexions pour une théorie des écosystèmes. Bull. Ecol., 8, 445-464.

FUJIKAWA H., USHIODA H., KUDO Y., 1992. Kinetics of Escherichia coli destruction by microwave irradiation. Appl. Environ. Microbiol., 58, 920-924.

HAVELAAR A.H., DURING M., WERSTEEGH J.F.M., 1987. Ampicillin-dextrin agar medium for the enumeration of Aeromonas species in water by membrane filtration. J. Appl. Bact. 62, 279-287.

HUMBEL F.X., 1969. Porosité, densité et perméabilité des sols ferralitiques rouges et jaunes près de Yaoundé. Document ORSTOM, Série sol, Yaoundé.

JAYAMOHAN S., OHGAKI S., HANAKI K., 1988. Effect of DO on kinetics of nitrification. Water Supply, 6, 141-150.

KAPER J.B., LOCKMAN H., COLWELL R.R., 1981. Aeromonas hydrophila: ecology and toxigenicity of isolates from an estuary. J. Appl. Bact., 50, 359-377.

KREISEL W., 1991. Water quality and health. Wat. Sci. Tech., 23, 201-209.

LARPENT J.-P., LARPENT-GOURGAUD M., 1990. Mémento technique de microbiologie, $2^{\mathrm{e}}$ édition, Tec \& Doc Lavoisier, Paris.

LE MINOR L., VÉRON M., 1989. Bactériologie médicale, $2^{\mathrm{e}}$ édition, Flammarion, Paris.

MARCHAL N., BOURDON J.L., RICHARD $\mathrm{Cl}_{1}, 1991$. Les milieux de culture pour l'isolement et l'identification biochimique des bactéries, $4^{\mathrm{e}}$ édition, Doin, Paris.
MARSHALL K.C., 1988. Adhesion and growth of bacteria at surfaces in oligotrophic habitats. Can. J. Microbiol., 34, 503-506.

MATTHEWS K. R., JAYARAO B.M., GUIDRY A.J., ERBE E.F., WERGIN W.P., OLIVIER S.P., 1994. Encapsulation of Streptococcus uberis: influence of storage and cultural condition. Vet. Microbiol., 39, 361-367.

NOLA M., NJINÉ T., BOUTIN C., 1998. Variabilité de la qualité des eaux souterraines dans quelques stations de Yaoundé (Cameroun). Mém. Biospéol., 25, 183-191.

NOLA M., NJINE T., MONKIEDJE A., TAILLEZ R., 1999. Approche colimétrique des eaux de la nappe phréatique superficielle de Yaoundé (Cameroun). Microbiol. Hyg. Alim., 11, 9-14.

NOLA M., NJINE T., DJUIKOM E., SIKATI FOKO V., 2000. Bacteria indicators dynamics in wells as influenced by well depth and well water column thickness, in Yaounde (Cameroon). Afr. J. Sci. Technol., 1, 82-91.

PELMONT J., 1993. Bactérie et environnement. Adaptations physiologiques. Presse universitaire de Grenoble, Grenoble.

RODIER J., 1996. L'analyse de l'eau, $8^{\mathrm{e}}$ édition, Dunod, Paris.

ROSZAK D.B., COLWELL R.R., 1987. Survival strategies of bacteria in the natural environment. Microbiol. Rev., 51, 365379.

SANDER B.C., KALFF J., 1993. Factors controlling bacterial production in marine and freshwater sediments. Microb. Ecol., 26, 79-99.

SEIDLER R.J., ALLEN D.A., LOCKMAN H., COLWELL R.R., JOSEPH S.W., DAILY O.P., 1980. Isolation, enumeration, and characterization of Aeromonas from polluted waters encountered in diving operations. Appl. Environ. Microbiol., 39, 1010-1018.

TSUJIHATA S., ENTANI E., ASAI M., TSUKAMOTO Y., OHTA M., 1998. Inactivation models for Salmonella typhimurium, Staphylococcus aureus and Vibrio parahaemolyticus in processed vinegar. Biocontrol Science, 3, 93-98.

TUNCAN E.U., 1993. Effect of cold temperature on germicidal efficacy of quaternary 
ammonium compound, iodophor, and chlorine on Listeria. J. Food Protection, 56, 1029-1033.

WIEBE W.J., SHELDON W.M., POMEROY L.R., 1992. Bacterial growth in the cold: evidence for an enhanced substrate requirement. Appl. Environ. Microbiol., 58, 359-364.

WILLOCX F., MERCIER M., HENDRICKX M., TOBBACK P., 1993. Modelling the influence of temperature and carbon dioxide upon the growth of Pseudomonas fluorescens. Food Microbiology, 10 , 159-173.

YONGUE-FOUATEU R., 1986. Contribution à l'étude pétrologique de l'altération et des faciès de cuirassement ferrugineux des gneiss migmatitiques de la région de Yaoundé. Th. Doct., Univ. Yaoundé, $214 \mathrm{p}$. 